\title{
SOME RESULTS FOR VACATION SYSTEMS WITH SOJOURN TIME LIMITS
}

\author{
TSUYOSHI KATAYAMA,* Chubu Teletraffic Engineering Laboratory
}

\begin{abstract}
In this paper we deal with an M/G/1 vacation system with the sojourn time (wait plus service) limit and two typical vacation rules, i.e. multiple and single vacation rules. Using the level crossing approach, explicit expressions for the steady-state distributions of the virtual waiting times are obtained in vacation systems with exponential and constant service times, a general vacation time, and two vacation rules.
\end{abstract}

Keywords: Sojourn time limit; vacation system; balking; level crossing; integral equation

2010 Mathematics Subject Classification: Primary 45D05; 60G17; 60K25

\section{Introduction}

Single-server queues with service vacations arise as models of many computer, communication, and production systems. A wide class of such vacation models has been studied extensively in the applied probability literature. We consider an M/G/1 vacation model with the sojourn time limit. An M/G/1 model with server vacations and limited sojourn time was studied in Takine and Hasegawa (1990) under a different rule for customers staying for service. A stochastic process of the unfinished work in an M/G/1 vacation model with finite capacity for the workload, which is equivalent to their model with limited sojourn time, was also studied in van der Duyn Schouten (1978). To the author's knowledge, only these two papers deal with the vacation model with the sojourn time limit. The single-server queue with limited sojourn time and constant service time was studied in Hokstad (1979); however, server vacations were not considered. Related references on the single-server queue with limited waiting time are provided in Takine and Hasegawa (1990).

Using the level crossing approach pioneered by Brill in 1974 (see Brill (2008)), we derive the steady-state probability density functions of the virtual waiting times for both the multiple and single vacation rules as described below. We describe our mathematical model in detail. Customers arrive at a queue according to a Poisson process with rate $\lambda$. They are served by a single server in order of arrival (first-in-first-out (FIFO) discipline), where the service time of a customer is denoted by $H$. They cannot stay in the system longer than an interval of length $T$. We assume that the sojourn times of arriving customers are known by a system manager, at their arrival instants, and they are refused entry to the system if their sojourn time $\Theta$ exceeds the interval $T$, i.e. $\Theta>T$. (The system manager may utilize a reneging rule before starting service for the rejected customers). If the queue becomes empty at the end of a service period, the server takes a vacation with vacation time $V$, (i.e. the so-called exhaustive service vacation).

Received 6 January 2010; revision received 10 April 2011.

* Postal address: Chubu Teletraffic Engineering Laboratory, Naka-taikouyama 4-66, Imizu-shi, Toyama, 939-0363, Japan. Email address: katagon@silk.plala.or.jp 
We consider the following two typical vacation rules.

(i) If the system is empty upon the server's return from a vacation, another vacation begins immediately. If the system is not empty upon the server's return, the server starts servicing again (multiple vacation (MV) rule).

(ii) If the system is empty upon the server's return from a vacation, the server does not take another vacation and waits for a new arrival. If the system is not empty upon the server's return, the server starts servicing again, i.e. the server takes exactly one vacation (single vacation (SV) rule).

The service time $H$ and the vacation time $V$ are independent and identically distributed random variables. Given a nonnegative-valued random variable $F$, we denote its distribution function (DF) by $F(t)$, its probability density function (PDF) by $f(t)$, its Laplace-Stieltjes transform (LST) by $F^{*}(s)$, and its finite first and $m$ th moments of the DF by $f$ and $f^{(m)}, m=$ $2,3, \ldots$, respectively. We define $\bar{F}(t):=1-F(t)$ for $t \geq 0$ and $\rho:=\lambda h$.

\section{Level crossing analysis}

We first consider a sample path (sample function) $W_{t}$ of the virtual workload process $\left\{W_{t}\right\}$ of the M/G/1 queueing system with sojourn time limit and vacation times, where $W_{t}$ represents the virtual workload $(W)$ at a time $t \geq 0$. The virtual workload is defined as the sum of the unfinished workload in the system and the remaining vacation time, that is, $W$ represents the virtual waiting time under the FIFO discipline. Figures 1 and 2 show typical sample paths of $\left\{W_{t}\right\}$ for the MV and the SV rules, respectively. The virtual workload process $\left\{W_{t}\right\}$ is a stochastic process with independent cycles defined as an interval between successive vacation starting epochs, in which the expected length of a cycle is denoted by $\mathrm{E}(C)$. Throughout this section, we assume that the system is in equilibrium. We denote by $W(x)$ the steady-state DF of the virtual waiting time $W$, and assume that $W(x)$ is differentiable for $x>0$. We formulate the level crossing formula (balance equation) in (4) and (5) below. We assume that the service time $H$ has an exponential distribution, i.e. $H(t)=1-\mathrm{e}^{-\mu t}$.

Theorem 1. For the $M / M / 1 M V$ system with limited sojourn time $(T), w(x), x>0$, and $W(0)$ are given by

$$
\begin{aligned}
w(x) & = \begin{cases}\lambda_{v} \omega_{0}(x), & x<T, \\
\lambda_{v} \bar{V}(x), & x \geq T,\end{cases} \\
\omega_{0}(x) & :=\rho\left(1-\mathrm{e}^{-\mu(T-x)}\right) \mathrm{e}^{-(\lambda-\mu)(T-x)} \exp \left\{-\rho \mathrm{e}^{-\mu(T-x)}\right\} \Psi(x)+\bar{V}(x), \\
\Psi(x) & :=\mu \mathrm{e}^{(\lambda-\mu) T} \int_{0}^{x} \mathrm{e}^{-(\lambda-\mu) y} \bar{V}(y) \exp \left\{-\rho \mathrm{e}^{-\mu(T-y)}\right\} \mathrm{d} y, \\
\lambda_{v} & :=\left(\int_{0+}^{T} \omega_{0}(x) \mathrm{d} x+\int_{T}^{\infty} \bar{V}(x) \mathrm{d} x\right)^{-1}, \\
W(0) & =0
\end{aligned}
$$

Proof. The level crossing formula is given by

$$
w(x)= \begin{cases}\lambda_{v} \bar{V}(x)+\lambda W(0)[\bar{H}(x)-\bar{H}(T)] & \\ \quad+\lambda \int_{0+}^{x}[\bar{H}(x-y)-\bar{H}(T-y)] w(y) \mathrm{d} y, & 0<x<T, \\ \lambda_{v} \bar{V}(x), & x \geq T .\end{cases}
$$




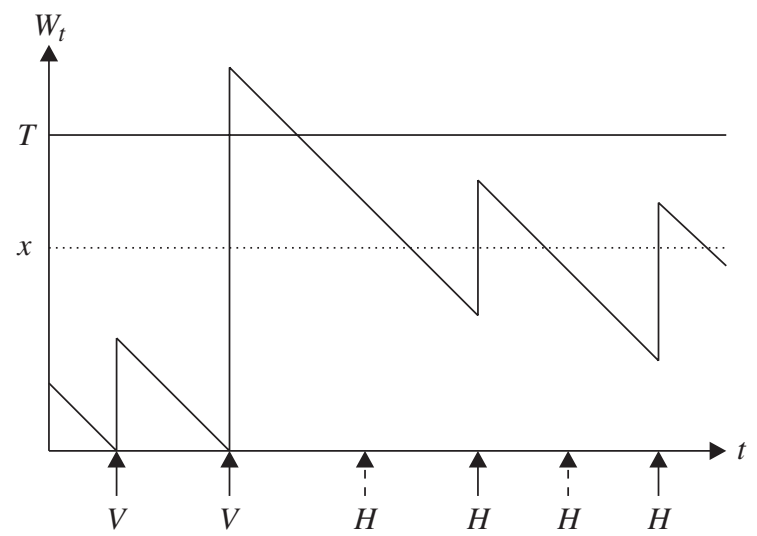

Figure 1: A sample path $W_{t}$ for the MV rule.

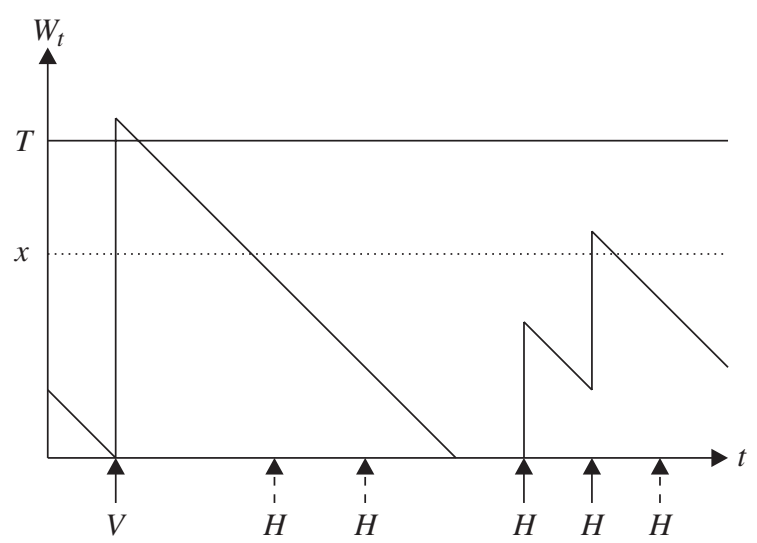

FIgURE 2: A sample path $W_{t}$ for the SV rule.

The left-hand side of (4) represents the rate at which $W_{t}$ downcrosses a level $x>0$, while the right-hand side represents the rate at which $W_{t}$ jumps from below $x$ to above $x>0$. The first term on the right-hand side of (4) corresponds to the case where the server takes vacations, i.e. a vacation (customer) brings to the system the workload $V$ and then $W_{t}$ jumps from below $x$ to above $x$. The second and third terms correspond to the case where an ordinary customer brings to the system the workload $H$ and then $W_{t}$ jumps from below $x$ to above $x$; see Figure 1 . The minus terms, $\bar{H}(T)$ and $\bar{H}(T-y)$, correspond to the case where arriving customers are rejected entry to the system because their sojourn time $\Theta=W+H>T$ (such arrivals with service time $H$ are shown in Figure 1 using dash-arrowed lines). This argument can also be applied to (5). Note here that $W(0)=0$ for the MV rule, and the arrival rate of vacation customers $\left(\lambda_{v}\right)$ is yet unknown. It follows from (4) and $\bar{H}(x)=\mathrm{e}^{-\mu x}$ that

$$
w(x)=\lambda_{v} \bar{V}(x)+\lambda\left(\mathrm{e}^{-\mu x}-\mathrm{e}^{-\mu T}\right) \int_{0+}^{x} \mathrm{e}^{\mu y} w(y) \mathrm{d} y .
$$

Setting $g_{0}^{\prime}(y):=\mathrm{e}^{\mu y} w(y)$, we obtain

$$
g_{0}^{\prime}(x)=\lambda_{v} \mathrm{e}^{\mu x} \bar{V}(x)+\lambda\left(1-\mathrm{e}^{\mu(x-T)}\right) g_{0}(x),
$$


where we have set $g_{0}(0+)=0$. Furthermore, setting $z:=\mathrm{e}^{-\mu(T-x)}$ and $g(z):=g_{0}(x)$, we obtain the following first-order differential equation for $g(z), 1>z>\mathrm{e}^{-\mu T}$ :

$$
g^{\prime}(z)+\rho\left(1-\frac{1}{z}\right) g(z)=q(z), \quad q(z):=\frac{\lambda v}{\mu} \mathrm{e}^{\mu T} \bar{V}\left(T+\frac{1}{\mu} \log z\right) .
$$

Taking account of the boundary condition $g_{0}(0+)=0$, the solution is expressed as

$$
g(z)=z^{\rho} \mathrm{e}^{-\rho z} \int_{\mathrm{e}^{-\mu T}}^{z} y^{-\rho} \mathrm{e}^{\rho y} q(y) \mathrm{d} y .
$$

Consequently, using the relation $w(x)=g_{0}^{\prime}(x) \mathrm{e}^{-\mu x}, 0<x<T$, we obtain (1) and (2), where $\lambda_{v}$ is yet unknown. For $x \geq T, w(x)$ is given by (5), directly. The arrival rate $\lambda_{v}$ of vacation customers should be determined from the normalization condition

$$
W(0)+\int_{0+}^{T} w(x) \mathrm{d} x+\int_{T}^{\infty} w(x) \mathrm{d} x=1,
$$

which concludes the proof.

Theorem 2. For the M/M/1 SV system with limited sojourn time $(T), w(x), x>0$, and $W(0)$ are given by

$$
\begin{aligned}
w(x)= & \begin{cases}\lambda_{v} \omega_{0}(x), & x<T \\
\lambda_{v} \bar{V}(x), & x \geq T\end{cases} \\
\omega_{0}(x):= & \rho\left(1-\mathrm{e}^{-\mu(T-x)}\right) \mathrm{e}^{-(\lambda-\mu)(T-x)} \exp \left\{-\rho \mathrm{e}^{-\mu(T-x)}\right\} \Psi(x)+\bar{V}(x) \\
& +q_{0}\left(\mathrm{e}^{-\mu x}-\mathrm{e}^{-\mu T}\right), \\
\Psi(x):= & \mu \mathrm{e}^{(\lambda-\mu) T} \int_{0}^{x} \mathrm{e}^{-(\lambda-\mu) y}\left[\bar{V}(y)+q_{0}\left(\mathrm{e}^{-\mu y}-\mathrm{e}^{-\mu T}\right)\right] \exp \left\{-\rho \mathrm{e}^{-\mu(T-y)}\right\} \mathrm{d} y, \\
q_{0}:= & V^{*}(\lambda), \\
\lambda_{v}:= & \left(\frac{q_{0}}{\lambda}+\int_{0+}^{T} \omega_{0}(x) \mathrm{d} x+\int_{T}^{\infty} \bar{V}(x) \mathrm{d} x\right)^{-1}, \\
W(0)= & \lambda_{v} \frac{q_{0}}{\lambda} .
\end{aligned}
$$

Proof. For the SV system, the level crossing formula is also given by (4) and (5). Furthermore, using the property of the regenerative process $\left\{W_{t}\right\}$ (see, e.g. Asmussen (2003, pp. 168171)), we have

$$
\lambda_{v}=\frac{1}{\mathrm{E}(C)}, \quad W(0)=\frac{(1 / \lambda) q_{0}+0 \times\left(1-q_{0}\right)}{\mathrm{E}(C)},
$$

where $1 / \lambda$ represents the mean nonbusy period, in which $W_{t}=0$ and $q_{0}$ is the probability that during a vacation no customers arrive; see Figure 2. Using the same argument as in the proof of Theorem 1, we obtain the following differential equation for $g(z), 1>z>\mathrm{e}^{-\mu T}$ :

$$
g^{\prime}(z)+\rho\left(1-\frac{1}{z}\right) g(z)=q(z), \quad q(z):=\frac{\lambda_{v}}{\mu}\left[\mathrm{e}^{\mu T} \bar{V}\left(T+\frac{1}{\mu} \log z\right)+q_{0}\left(\frac{1}{z}-1\right)\right] .
$$

Hence, $\lambda_{v}$ is given by (7), which follows from (6), (9), and the same argument as in the proof of Theorem 1. Equation (9) leads to (8). 
We assume hereafter that $H$ is constant, i.e. $H(t)=0(t<h)$ and $H(t)=1(t \geq h)$.

Theorem 3. For the $M / D / 1 M V$ system with limited sojourn time $(T), w(x), x>0$, and $W(0)$ are given by

$$
w(x)=\left\{\begin{array}{l}
\lambda_{v} \omega_{k}(x) \quad \text { for } x \in I_{k}, k=0,1, \ldots, n+1, x<T, \\
\lambda_{v} \bar{V}(x) \quad \text { for } x \geq T,
\end{array}\right.
$$

where the $I_{k}, k=0,1, \ldots, n+1(n \geq 1)$, are given in (16) and (17) below, the $\omega_{k}(x), k=$ $0,1, \ldots, n+1$, are defined by

$$
\begin{aligned}
\omega_{k}(x) & :=\bar{V}(x)+\lambda\left[\varphi_{k}(x)-\varphi_{k-1}(x-h)\right], \quad k=0,1, \ldots, n-1, \\
\omega_{n}(x) & :=\bar{V}(x)+\lambda\left[\varphi_{n-1}(T-h)-\varphi_{n-2}(x-h)\right], \\
\omega_{n+1}(x) & :=\bar{V}(x)+\lambda\left[\varphi_{n-1}(T-h)-\varphi_{n-1}(x-h)\right],
\end{aligned}
$$

with the $\varphi_{k}(x), k=0,1, \ldots, n-1$, recursively obtained from (22) below and $\varphi_{-1}(x) \equiv 0$, and

$$
\begin{aligned}
\lambda_{v} & :=\left(\varphi_{n-1}(T-h)+\int_{T-h}^{n h} \omega_{n}(x) \mathrm{d} x+\int_{n h}^{T} \omega_{n+1}(x) \mathrm{d} x+\int_{T}^{\infty} \bar{V}(x) \mathrm{d} x\right)^{-1}, \\
W(0) & =0 .
\end{aligned}
$$

Proof. For the M/D/1 vacation system (MV), the level crossing formula is given by (4) and (5). To obtain the solution of (4) and (5), we start by dividing [0,T) into $n+2$ subintervals, i.e.

$$
\begin{aligned}
I_{k} & :=\{x: k h \leq x<(k+1) h\}, \quad k=0,1, \ldots, n-2, \\
I_{n-1} & :=\{x:(n-1) h \leq x<T-h\}, \\
I_{n} & :=\{x: T-h \leq x<n h\}, \\
I_{n+1} & :=\{x: n h \leq x<T\},
\end{aligned}
$$

where $n$ is an integer satisfying

$$
n h \leq T<(n+1) h .
$$

Here the $n=0$ case is of no interest. The $n=1$ case requires a separate treatment, so we first assume that $n \geq 2$. Now we introduce

$$
W_{k}(x):=W(x) \text { for } x \in I_{k}, k=0,1, \ldots, n+1,
$$

and let $w_{k}(x)$ be the value of $w(x)$ for $x \in I_{k}$. Equation (4) gives, for $x \in I_{0}$,

$$
w_{0}(x)=\lambda_{v} \bar{V}(x)+\lambda W(0)+\lambda W_{0}(x),
$$

that is,

$$
W_{0}(x)=\lambda_{v} \varphi_{0}(x), \quad \varphi_{0}(x):=\mathrm{e}^{\lambda x} \int_{0}^{x} \mathrm{e}^{-\lambda y} \bar{V}(y) \mathrm{d} y,
$$

where we have used the fact that $W(0)=0$ for the MV rule. From (4) we consecutively obtain the following first-order differential equation for $W_{k}(x), x \in I_{k}, k=1,2, \ldots, n-1$ :

$$
\begin{aligned}
w_{k}(x) & =\lambda_{v} \bar{V}(x)+\lambda\left[W_{k}(x)-W_{k-1}(x-h)\right] \\
& =\lambda_{v} \bar{V}(x)+\lambda\left[W_{k}(x)-\lambda_{v} \varphi_{k-1}(x-h)\right],
\end{aligned}
$$


that is,

$$
\frac{\mathrm{d} W_{k}(x)}{\mathrm{d} x}-\lambda W_{k}(x)=\lambda_{v}\left[\bar{V}(x)-\lambda \varphi_{k-1}(x-h)\right] .
$$

Using the boundary condition $W_{k}(k h)=W_{k-1}(k h)$, the solution is given by

$$
\begin{aligned}
W_{k}(x) & =\lambda_{v} \varphi_{k}(x), \quad k=1,2, \ldots, n-1, \\
\varphi_{k}(x) & =\mathrm{e}^{\lambda(x-k h)} \varphi_{k-1}(k h)+\mathrm{e}^{\lambda x} \int_{k h}^{x} \mathrm{e}^{-\lambda y}\left[\bar{V}(y)-\lambda \varphi_{k-1}(y-h)\right] \mathrm{d} y .
\end{aligned}
$$

Furthermore, from (4) we obtain

$$
w_{n}(x)=\lambda_{v} \bar{V}(x)+\lambda\left[W_{n-1}(T-h)-W_{n-2}(x-h)\right]=\lambda_{v} \varphi_{n}(x), \quad x \in I_{n},
$$

where

$$
\varphi_{n}(x):=\bar{V}(x)+\lambda\left[\varphi_{n-1}(T-h)-\varphi_{n-2}(x-h)\right],
$$

and

$$
w_{n+1}(x)=\lambda_{v} \bar{V}(x)+\lambda\left[W_{n-1}(T-h)-W_{n-1}(x-h)\right]=\lambda_{v} \varphi_{n+1}(x), \quad x \in I_{n+1},
$$

where

$$
\varphi_{n+1}(x):=\bar{V}(x)+\lambda\left[\varphi_{n-1}(T-h)-\varphi_{n-1}(x-h)\right] .
$$

Equations (11), (12), and (13) are obtained from (20), (23), and (24), respectively.

We have considered only the $n \geq 2$ case in (16) and (17). It can also be shown that the above results hold even for $n=1$, by dividing the interval $[0, T)$ into three subintervals, $I_{0}:=[0, T-h), I_{1}:=[T-h, h)$, and $I_{2}:=[h, T)$, and setting $\varphi_{-1}(x) \equiv 0$, which is obtained from (19) and (22). Furthermore, for $x \geq T, w(x)$ is given by (5), directly. The arrival rate $\lambda_{v}$ should be determined from the normalization condition

$$
W_{n-1}(T-h)+\int_{T-h}^{n h} w_{n}(x) \mathrm{d} x+\int_{n h}^{T} w_{n+1}(x) \mathrm{d} x+\int_{T}^{\infty} w(x) \mathrm{d} x=1,
$$

which leads to (14).

Theorem 4. For the M/D/I SV system with limited sojourn time $(T), w(x), x>0$, and $W(0)$ are given by

$$
w(x)=\left\{\begin{array}{l}
\lambda_{v} \omega_{k}(x) \quad \text { for } x \in I_{k}, k=0,1, \ldots, n+1, x<T, \\
\lambda_{v} \bar{V}(x) \quad \text { for } x \geq T,
\end{array}\right.
$$

where the $I_{k}, k=0,1, \ldots, n+1(n \geq 1)$, are given in (16) and (17), the $\omega_{k}(x), k=$ $0,1, \ldots, n+1$, are defined by

$$
\begin{aligned}
\omega_{k}(x) & :=\bar{V}(x)+\lambda\left[\varphi_{k}(x)-\varphi_{k-1}(x-h)\right], \quad k=0,1, \ldots, n-1, \\
\omega_{n}(x) & :=\bar{V}(x)+\lambda\left[\varphi_{n-1}(T-h)-\varphi_{n-2}(x-h)\right], \\
\omega_{n+1}(x) & :=\bar{V}(x)+\lambda\left[\varphi_{n-1}(T-h)-\varphi_{n-1}(x-h)\right],
\end{aligned}
$$

with the $\varphi_{k}(x), k=0,1, \ldots, n-1$, recursively obtained from (22) and $\varphi_{-1}(x) \equiv q_{0} / \lambda, q_{0}:=$ $V^{*}(\lambda)$, and

$$
\begin{aligned}
\lambda_{v} & :=\left(\varphi_{n-1}(T-h)+\int_{T-h}^{n h} \omega_{n}(x) \mathrm{d} x+\int_{n h}^{T} \omega_{n+1}(x) \mathrm{d} x+\int_{T}^{\infty} \bar{V}(x) \mathrm{d} x\right)^{-1}, \\
W(0) & =\lambda_{v} \frac{q_{0}}{\lambda} .
\end{aligned}
$$


Proof. For the M/D/1 vacation system (SV), the level crossing formula is also given by (4) and (5). Using $I_{k}, k=0,1, \ldots, n+1$, for [0,T) defined by (16) together with (17), we obtain the solution of (4), $w_{k}(x)$, and $W_{k}(x)$ defined in (18). First we consider the $n \geq 2$ case as in the proof of Theorem 3. Equation (4) gives, for $x \in I_{0}$,

$$
w_{0}(x)=\lambda_{v} \bar{V}(x)+\lambda W(0)+\lambda\left[W_{0}(x)-W_{0}(0+)\right],
$$

that is,

$$
W_{0}(x)=\lambda_{v} \varphi_{0}(x), \quad \varphi_{0}(x):=\mathrm{e}^{\lambda x}\left(\frac{q_{0}}{\lambda}+\int_{0}^{x} \mathrm{e}^{-\lambda y}\left[\bar{V}(y)-q_{0}\right] \mathrm{d} y\right),
$$

where we have used the fact that $W(0)=W_{0}(0+)=\lambda_{v} q_{0} / \lambda$, which is derived from (9) for the SV rule. As shown in the proof of Theorem 3, we obtain (21) together with (22), (23), and (24). Equations (27), (28), and (29) are obtained from (20), (23), and (24), respectively.

It can be shown that the above results hold for $n=1$, by dividing the interval $[0, T)$ into three subintervals, $I_{0}:=[0, T-h), I_{1}:=[T-h, h)$, and $I_{2}:=[h, T)$, and setting $\varphi_{-1}(x) \equiv q_{0} / \lambda$, which is obtained from (22) and (32). For $x \geq T, w(x)$ is given by (5). Finally, $\lambda_{v}$ in (30) is obtained from (25).

Remark 1. The functions $W(x)$ and $w(x)$ exist even for $1<\rho+\rho_{v}\left(:=\lambda_{v} v\right)<\infty$. (Such a result has been found for the single-server queue with waiting time limit in Takács (1974)). Note that all customers without rejection are guaranteed their service completion, that is, they wait, receive full service, and depart before their sojourn times reach $T$. It can be seen from the $W(x)$ and $w(x)$ formulae that the $\mathrm{M} / \mathrm{D} / 1$ vacation system analyzed in this paper is equivalent to the vacation system with waiting time limit $T-h$. Hence, $w(x)$ for the MV rule, i.e. (10), gives a different expression from $w(x)$ derived in Takine and Hasegawa (1990).

\section{Performance analysis}

Using Theorems 1-4 and the PASTA (Poisson Arrivals See Time Averages) property, the following results for an arbitrary customer are obtained for the MV rule and the SV rule. Let $p$ denote the blocking probability (or loss probability) that a customer cannot enter the system upon arrival.

Corollary 1. The blocking probability in the $M / M / 1$ vacation system is given by

$$
p= \begin{cases}\lambda_{v}\left(\mathrm{e}^{-\mu T} \int_{0+}^{T} \mathrm{e}^{\mu x} \omega_{0}(x) \mathrm{d} x+\int_{T}^{\infty} \bar{V}(x) \mathrm{d} x\right) & \text { for the MV rule, } \\ \lambda_{v}\left(\frac{q_{0}}{\lambda} \mathrm{e}^{-\mu T}+\mathrm{e}^{-\mu T} \int_{0+}^{T} \mathrm{e}^{\mu x} \omega_{0}(x) \mathrm{d} x+\int_{T}^{\infty} \bar{V}(x) \mathrm{d} x\right) & \text { for the } S V \text { rule, }\end{cases}
$$

where $\lambda_{v}$ and $\omega_{0}(x)$ are given in (2) for the $M V$ rule and (7) for the SV rule. In the $M / D / 1$ vacation system,

$$
\begin{array}{r}
p=\lambda v\left(\rho \varphi_{n-1}(T-h)+\int_{T-h}^{\infty} \bar{V}(x) \mathrm{d} x-\lambda \int_{T-h}^{n h} \varphi_{n-2}(x-h) \mathrm{d} x\right. \\
\left.-\lambda \int_{n h}^{T} \varphi_{n-1}(x-h) \mathrm{d} x\right) \text { for both the } M V \text { and } S V \text { rules }
\end{array}
$$

where $n \geq 1$ is the integer satisfying $n h \leq T<(n+1) h$. Here the $\varphi_{m}(x), m=0,1, \ldots, n-1$, 
are recursively obtained from (22), where $\varphi_{-1}(x) \equiv 0$ (MV rule) and $\varphi_{-1}(x) \equiv q_{0} / \lambda(S V$ rule), and the $\lambda_{v}$ are given by (14) for the $M V$ rule and (30) for the $S V$ rule. The probability of no delay for a customer without blocking is given by

$$
W(0 \mid \text { no blocking })= \begin{cases}\frac{W(0)\left\{1-\mathrm{e}^{-\mu T}\right\}}{1-p} & \text { for the } M / M / 1 \text { vacation system, } \\ \frac{W(0)}{1-p} & \text { for the } M / D / 1 \text { vacation system, }\end{cases}
$$

where $W(0)=0$ for the $M V$ rule. For the $S V$ rule, $W(0)$ is as given in (8) (M/M/1 vacation system) and (31) (M/D/1 vacation system).

Proof. By definition we have

$$
p=\frac{1}{\lambda}\left(\lambda W(0) \bar{H}(T)+\lambda \int_{0+}^{T} \bar{H}(T-y) w(y) \mathrm{d} y+\lambda \int_{T}^{\infty} w(x) \mathrm{d} x\right),
$$

which leads to (33)-(34). The conditional probability of no delay for a customer without blocking is given by

$$
W(0 \mid \text { no blocking })=\frac{W(0) H(T)}{1-p},
$$

which leads to (35).

Corollary 2. For the $M / M / 1$ vacation system, the mth moment, $m=1,2, \ldots$, of the conditional DF of the actual waiting time for a customer without blocking is given by

$$
\mathrm{E}\left(W^{m} \mid \text { no blocking }\right)=\frac{\lambda_{v}}{1-p} \int_{0+}^{T} x^{m}\left(1-\mathrm{e}^{-\mu(T-x)}\right) \omega_{0}(x) \mathrm{d} x,
$$

where $\lambda_{v}$ and $\omega_{0}(x)$ are given in (2) for the MV rule and (7) for the SV rule. For the $M / D / 1$ vacation system,

$$
\begin{aligned}
\mathrm{E}\left(W^{m} \mid \text { no blocking }\right)=\frac{\lambda v}{1-p}\left(\sum_{k=0}^{n-2} \int_{k h}^{(k+1) h} x^{m}\left[\bar{V}(x)+\lambda \varphi_{k}(x)-\lambda \varphi_{k-1}(x-h)\right] \mathrm{d} x\right. \\
\left.+\int_{(n-1) h}^{T-h} x^{m}\left[\bar{V}(x)+\lambda \varphi_{n-1}(x)-\lambda \varphi_{n-2}(x-h)\right] \mathrm{d} x\right),
\end{aligned}
$$

where the $\varphi_{m}(x), m=0,1, \ldots, n-1$, are recursively obtained from (22), $\varphi_{-1}(x) \equiv 0(M V$ rule) and $\varphi_{-1}(x) \equiv q_{0} / \lambda$ (SV rule), and the $\lambda_{v}$ are given by (14) for the MV rule and (30) for the $S V$ rule. The empty sum $\sum_{k=0}^{-1}\{\cdot\} \equiv 0$.

Proof. By definition we have

$$
\mathrm{E}\left(W^{m} \mid \text { no blocking }\right)=\frac{1}{1-p} \int_{0}^{T} x^{m} H(T-x) \mathrm{d} W(x),
$$

which leads to (36) and (37). 


\section{Concluding remarks}

For the M/M/1 vacation systems (MV and SV rules) with a general vacation time distribution and sojourn time limit, explicit expressions for the PDFs of the virtual waiting times have been derived using the level crossing approach. For the M/D/1 vacation systems (MV and SV rules), recursive equations have been derived for the PDFs of the virtual waiting times. The level crossing formula given in (4) and (5) holds for the M/G/1 vacation system with sojourn time limit. An explicit solution of the Volterra integral equations is an open problem for more general service time distributions, though the level crossing formula for the M/G/1 vacation system (MV rule) with limited waiting time has been solved explicitly.

\section{References}

Asmussen, S. (2003). Applied Probability and Queues (Appl. Math. 51), 2nd edn. Springer, New York.

Brill, P. H. (2008). Level Crossing Methods in Stochastic Models (Internat. Ser. Operat. Res. Manag. Sci. 123). Springer, New York.

Hokstad, P. (1979). A single-server queue with constant service time and restricted accessibility. Manag. Sci. 25, 205-208.

TAKács, L. (1974). A single-server queue with limited virtual waiting time. J. Appl. Prob. 11, 612-617.

Takine, T. And Hasegawa, T. (1990). A note on M/G/1 vacation systems with waiting time limits. Adv. Appl. Prob. 22, 513-518.

VAN DER Duyn Schouten, F. A. (1978). An M/G/1 queueing model with vacation times. Z. Operat. Res. 22, 95-105. 